\title{
Successful Uterovaginal Anastomosis in an Unusual Presentation of Congenital Absence of Cervix
}

\author{
${ }^{1}$ Nusrat Mahmud, ${ }^{2}$ Naushaba Tarannum Mahtab, ${ }^{3}$ TA Chowdhury, ${ }^{4}$ Anjan Kumar Deb
}

\section{ABSTRACT}

Cervical agenesis or dysgenesis (fragmentation, fibrous cord and obstruction) is an extremely rare congenital anomaly. Conservative surgical approach to these patients involves uterovaginal anastomosis, cervical canalization and cervical reconstruction. In failed conservative surgery, total hysterectomy is the treatment of choice. We report what we believe to be the first successful end-to-end uterovaginal anastomosis of an unusual case of congenital cervical agenesis. A 25-yearold female presented complaining of primary amenorrhea and primary subfertility for the same duration. At laparoscopy, complete separation between the cervix and the body of the uterus was found and hanging from surrounding supports. Both ovaries and fallopian tubes were anatomically positioned. There was another muscular tissue of $2 \mathrm{~cm}$ in diameter at the pouch of Douglas which was attached with lateral pelvic wall by transverse cervical ligament. Upon readmission, laparotomy was performed under general anesthesia in a semilithotomy position allowing both abdominal and vaginal approaches. Uterovaginal anastomosis was done by the restoration of the genital tract by direct suturing of the isthmus uteri to the vagina. Insertion of a $16 \mathrm{~F}$ Foley catheter transvaginally through the vaginal opening into the endometrial cavity and was inflated with $5 \mathrm{ml}$ of fluid into the catheter balloon. There were no postoperative complications. Foley catheter was kept in situ for 21 days. Patient had her first menstruation on 11th day of surgery and that was continued for 5 days with regular flow. Consecutive 3-cycles follow-up has been done and the patient is having her normal menstruation.

In the presence of cervical anomaly with functional uterus and intact vagina, uterovaginal anastomosis is feasible and effective and should be applied as a first-line treatment option.

Keywords: Cervical fragmentation, Cervical agenesis, Cervical aplasia, Uterine malformation, Uterine reconstruction, Primary amenorrhea, Reconstruction of vagina.

How to cite this article: Mahmud N, Mahtab NT, Chowdhury TA, Deb AK. Successful Uterovaginal Anastomosis in an Unusual Presentation of Congenital Absence of Cervix. J South Asian Feder Menopause Soc 2014;2(2):105-110.

\footnotetext{
${ }^{1}$ Consultant, ${ }^{2}$ Senior Medical Officer, ${ }^{3,4}$ Chief Consultant

${ }^{1,3}$ Centre for Assisted Reproduction, BIRDEM, General Hospital Dhaka, Bangladesh

${ }^{2}$ Department of Obstetrics and Gynecology, BIRDEM, General Hospital, Dhaka, Bangladesh

${ }^{4}$ Department of Reconstructive Surgery, BIRDEM, General Hospital, Dhaka, Bangladesh

Corresponding Author: Nusrat Mahmud, Consultant, Centre for Assisted Reproduction, BIRDEM, Dhaka, Bangladesh, e-mail: nusratmahmud18@gmail.com
}

\section{Source of support: Nil}

Conflict of interest: None

\section{INTRODUCTION}

Primary amenorrhea is defined as absence of menstruation by the age of 14 years in the absence of secondary sex characteristics or the absence of periods by the age of 16 years regardless of appearance of secondary sex characters. In our last study, a series of total 108 cases of primary amenorrhea were reviewed. It was found that $69.4 \%$ were due Müllerian dysgenesis, $19.4 \%$ due to gonadal dysgenesis, $2.7 \%$ male pseudohermaphroditism and $2.7 \%$ due to genital tuberculosis. ${ }^{13}$

Alterations in the normal sequence of development of Mullerian duct lead to a wide spectrum of reproductive anomalies. Uterine malformations can be observed in 7 to $10 \%$ of all women and major malformations are seen in 2 to $3 \%$ of fertile women, 3\% of infertile women and 5 to $10 \%$ of those with repeated miscarriages. A rare form of abnormality involving only a part of the Müllerian duct leading to functioning uterus and cervical agenesis is also seen. ${ }^{1}$

Congenital absence of the cervix is a rare condition and occurs in one in 80,000 to 100,000 births. It is known to be associated with both partial and complete vaginal aplasia and renal anomalies. ${ }^{11}$ According to American Fertility Society, cervical agenesis should be classified as a type $1 b$ Müllerian anomaly. ${ }^{2}$ Presentation is usually with primary amenorrhea and cyclical lower abdominal pain. Endometriosis or pelvic infection may result from chronic hematometra. In recent retrospective review of 18 patients, $39 \%$ had associated vaginal aplasia. ${ }^{3}$

The established role of the cervix is to supply cervical mucus for sperm transport, to serve as a barrier to ascending infection and to maintain a pregnancy in utero.

Recent literature, however, have established that in the absence of the cervix, surgical uterovaginal communication affords the possibility of using advanced reproductive technologies to achieve a pregnancy. A cerclage of lower uterine segment was required for some patients following uterovaginal anastomosis. Most series have reported delivery by cesarean section. Obstetrical complications have not been fully reported. ${ }^{12}$ 


\section{Anatomic Variations of Congenital Cervical Anomalies}

Two basic categories of cervical anomalies have been observed in several configurations. Patients exhibiting the first type, cervical aplasia, lack a uterine cervix (Figs 1A to C), ${ }^{3}$ and the lower uterine segment narrows to terminate in a peritoneal sleeve at a point well above the normal communication with the vaginal apex. The second type, cervical dysgenesis, can be described as four subtypes:

1. Cervical body consisting of a fibrous band of variable length and diameter (endocervical glands may be noted on pathologic examination; Fig. 1A).

2. Intact cervical body with obstruction of the cervical os (cervical ostium; the cervix is usually well formed, but a portion of the endocervical lumen is obliterated; Figs $1 B$ and $C$ ).

3. Stricture of the mid portion of the cervix (which is hypoplastic with a bulbous tip and no identifiable cervical lumen; Fig. 1C).

4. Fragmentation of the cervix (with portions that can be palpated below the fundus and that are not connected to the lower uterine segment; Fig. 1D).

Associated anomalies of the urinary tract are rare, but they do occur. Variable portions of the vagina can be atretic. Cervical obstruction is most often associated with a vagina of normal length.

\section{CASE REPORT}

We report the case of a 25 years old married female with the history of primary subfertility for 5 years and secondary amenorrhea for the same duration. She gives a history of regular menstruation prior to her marriage and she succumbed to a road traffic accident 2 months before her marriage and since then she developed secondary amenorrhea. She was prescribed progesterone and oral pills but no withdrawal bleeding occured. On examination, she had normal secondary sex characteristics. There was no significant medical or surgical history. There was no scar mark in the body which supports RTA. She did not give any history of dysparunea or coital dysfunction. During initial investigation at the referring center, all routine blood tests and hormone profiles were done and revealed no abnormality. Ultrasound of the lower abdomen showed normal size ovaries, normal size of the uterus with abrupt narrowing at the uterocervical junction with endometrial thickness of $11.1 \mathrm{~mm}$. A provisional diagnosis of primary subfertilty with secondary amenorrhea due to Asherman's syndrome was made and we planned for hysteroscopy and laparoscopy. When patient was examined under anesthesia, hysteroscope was not possible to introduce beyond the internal os. Dilatation was tried by Hagers dilator and it was possible to introduce only $1 \mathrm{~cm}$ above the internal os and a blind vagina was found. We abundant the hysteroscopy procedure and decided to go for laparoscopy.

A single intravenous dose of antibiotic was given with induction of anesthesia. The patient was prepared for surgery and cleaned and draped. She was initially placed supine without any table tilt. The legs were placed in a lithotomy position at an angle of $30^{\circ}$ at the hips. Bladder was evacuated by metallic catheter. An incision was made in the umbilical region. A Verres needle (Ethicon, Cincinnati, $\mathrm{OH}$ ) was used to obtain a pneumoperitoneum. The needle first was directed toward the sacral promontory, and when the characteristic clicks of passing through the layers were felt, the needle then was directed toward the pelvis. A water test was used to confirm entry into the correct space, and the abdomen was insufflated. Insufflation was ceased when flow ceased as a result of the preset pressure of $20 \mathrm{~mm} \mathrm{Hg}$. The main $10 \mathrm{~mm}$ trocar then was placed through the umbilical region.

Once the scope was inserted, the abdominal contents were examined, and the patient was placed in a headdown tilt. All additional ports were placed under direct
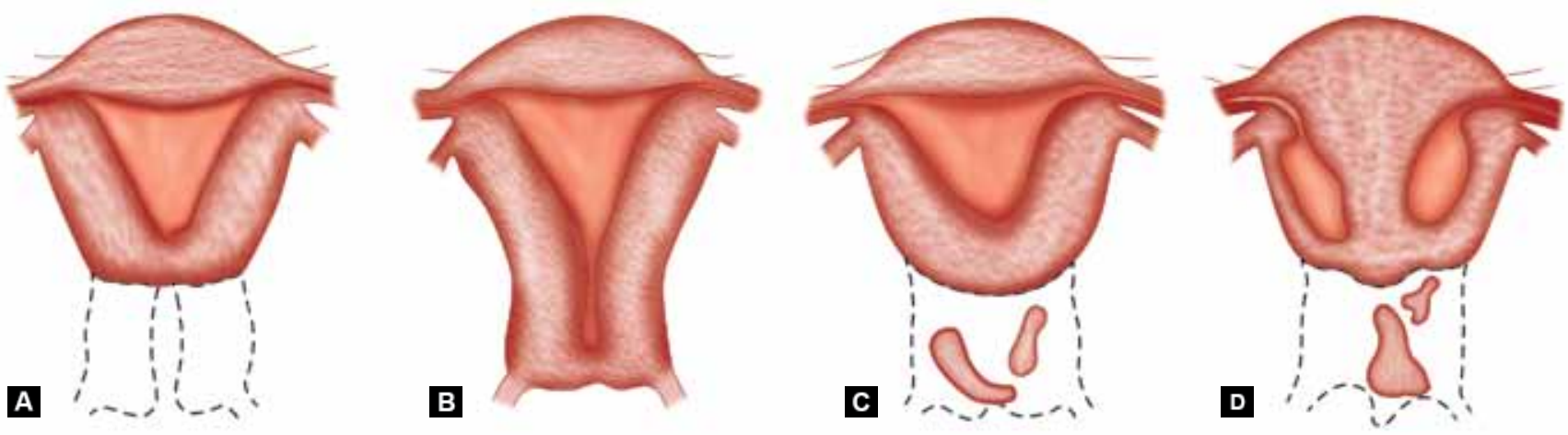

Figs 1A to D: Variations in cervical dysgenesis: (A) The fundus of the uterus is noted without a cervix, (B) the cervical body is intact with obstruction of the cervical os (cervical ostium), (C and $D)$ fragmentation of the cervix in which portions of the cervix are noted with no connection of the uterine body. In one patient, hypoplasia of the uterine cavity was noted ${ }^{3}$ 
vision to avoid injury to viscera or vessels. We placed two lateral $5 \mathrm{~mm}$ ports. The lateral ports were placed $2 \mathrm{~cm}$ above and medial to anterior superior iliac spine parallel to inguinal ligaments.

On introduction of the telescope within the abdomen (Figs 2A to F), it was found that there was complete separation between the cervix and the body of the uterus. Uterus was completely closed at the lower part of the body. It was freely mobile and hanging from surrounding supports. Both ovaries were symmetrically attached to the cornue of the uterus. Apart from the inflammatory changes on the surface of the body, rest of the fundus and body were normal in size and shape. Both the fallopian tubes were normal with normal attachments. There was another muscular tissue of $2 \mathrm{~cm}$ in diameter at the pouch of Douglas which was attached with lateral pelvic wall by transverse cervical ligament. Liver, gallbladder was normal. No other congenital anomaly could be found. Abdominal ports were closed with nonabsorbable 2-0 vicryl suture in tapered needle. Patient made an unremarkable recovery. Stitches were removed at 7 th postoperative day.

We have discussed with our patient and her husband with each and every possible surgical modalities of


Figs 2A to F: Laparoscopic views: (A) Panaromic view showing the complete separation of uterine body and cervix, (B) showing the lower end of body of the uterus, $(C)$ longitudinal view showing a gap in the vesicouterine reflection of peritoneum, (D) view from reverse direction. Lower end of the body with bilateral normal ovaries, $(E)$ cervical tissue and $(F)$ uterosacral ligament attached with the cervical tissue 
her treatment and possible outcome. They opted for uterovaginal anostomosis and accordingly we consulted with our plastic surgery colleague and laparotomy was decided.

Upon readmission, initial laparoscopy confirmed the above findings. Laparotomy was then performed under general anesthesia in a semilithotomy position allowing both abdominal and vaginal approaches. At dissection of the anterior space (between bladder and the uterus) cervix was found, totally, disrupted from the uterus. Distal cervical part was in place attached to the vagina with Mackenrot and uterosacral ligaments. Corpus uteri, isthmus uteri were detached from the distal part of the cervix within the broad ligament in a medial plane.

A surgical end-to-end anastomosis was decided upon and performed in the following steps (Figs 3 and 4): (i) surgical anatomical preparation of lower end of uterus and vagina, (ii) insertion of a silicon 16F Foley catheter transvaginally through the vaginal opening and ballooning of the catheter into the endometrial cavity with $3 \mathrm{ml}$ of fluid, (iii) suturing of vaginal edges to each other with the use of Vicryl absorbable suture No. 3.0, (iv) suturing of anterior and posterior uterovaginal/rectovaginal vault. The procedure was uneventful. Antibiotics were adminis- tered postoperatively. The Foley catheter remained in uterine cavity for 21 days. There were no postoperative complications. Patient had her first menstruation on 11th day of surgery and that was continued for 5 days with regular flow. Consecutive 3-cycle follow-up has been done and patient is having her normal menstruation.

\section{Key Points of Treatment of Cervical Dysgenesis}

- Hysterectomy is recommended for the patients with complete cervical agenesis.

- High uterovaginal graft, at the line of the endometrium in the lower uterine segment, may produce a more successful uterovaginal anastomosis. There is a high risk of stenosis and fibrosis at the anastomosis site.

- Cervicovaginal anastomosis may be accomplished in the patients with an endocervical canal. Cervicovaginal anastomosis and cervical grafting may be required.

- When necessary, the surgeon should be prepared to perform a vaginoplasty, whether or not the uterus is preserved.

- The anatomical findings in the cervix and patient preferences must be used for a comprehensive treatment strategy to afford the most successful outcome.


Figs 3A to C: Steps of laparotomy: (A) Complete uterovaginal dysjunction, (B) dilator passing through posterior fornix and (C) $3 \mathrm{ml}$ balloon was kept inflated inside the uterine cavity through an incision on the posterior uterine wall
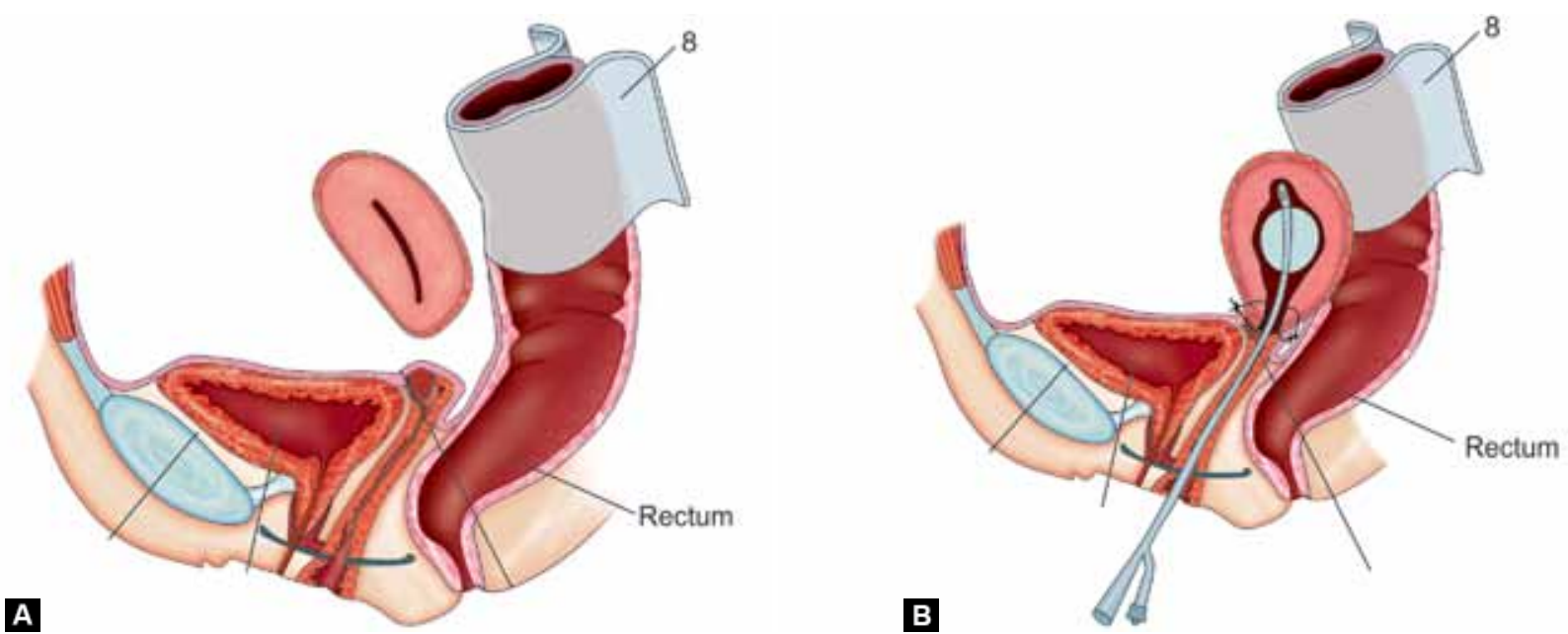

Figs 4A and B: Steps of laparotomy: (A) Floating uterus separated from the cervix and

(B) final postoperative state 
- Complications following uterovaginal anastomosis are not insignificant and include endometritis, pelvic inflammatory disease, persistent pelvic pain, bowel and bladder injury, reobstruction and stenosis requiring reoperation and death.

\section{DISCUSSION}

Müllerian duct anomalies, although rare, present in multiple ways from infancy to young adulthood. Female patients may present with mucocolpos, hematocolpos or hematometra, primary amenorrhea, pelvic pain, repeated pregnancy loss and infertility. One of the rarest congenital anomalies is cervical agenesis as the absence of the cervix. These patients present with amenorrhea, debilitating pelvic pain and almost commonly have a large pelvic mass. The incidence of cervical agenesis is unknown, but a review of the literature lists less than 200 cases since 1942.

The uterine cervix provides a conduct for menstrual flow, maintenance of an intrauterine pregnancy, mucus for sperm transfer, and a barrier to infection from vaginal microflora. Atresia (dysgenesis) of the cervix may result from local segmental atrophy.

Buttrum ${ }^{10}$ has suggested a classification for 'Müllerian agenesis or hypoplasia. Class I defects consist of a variety of malformations. Cervical anomalies are designated Ib. Our findings suggest that this classification should be broadened to include patients with the various forms of cervical dysgenesis. The presence or absence of a normal vagina should also be taken into considerations.

There is a consensus in the international literature that hysterectomy is the procedure of choice in patient with cervical dysgenesis. ${ }^{5}$ Alternately, when cervical dysgenesis is noted, reconstruction of cervix may be warranted. ${ }^{4}$

The goals of reconstructive surgery for cervical malformation are to provide a conduit for menstruation to relieve pain and preserve reproductive potential. The goals are usually achieved when there is substance to the cervix. Moreover, pregnancy has been documented after cervical reconstruction when cervical stroma is substantial. ${ }^{6}$

In our patient, there was complete separation of cervix without any stroma. Our patient did not give any history of cyclical pain, so the option for hysterectomy was abundant. Psychological stress and marital disharmony is an extra burden for these patients. Sometimes they make up twisted history which exactly happened in our patient of having a road traffic accident.

Anastomosis of the cervix to the uterine body may be accomplished with success, provided the distal cervical segment is well formed as that was not in our case. Uterovaginal anastomosis can be a good option for these patients. The best surgical approach requires a discussion on the postoperative complications, the degree of cervical abnormality and patient's desired treatment outcome. In cases of cervical agenesis or cervical dysgenesis, where the cervix is absent or there is no functional cervical part (cervical fragmentation/fibrous cord), uterovaginal anastomosis and cervical reconstruction (creation of neocervix) have been proposed as treatment options. In patients with coexistent vaginal aplasia, the creation of a neovagina is necessary. Uterovaginal anastomosis is the restoration of the genital tract by direct suturing of the isthmus uteri to the vagina. Cervical reconstruction is, practically, an attempt to restore the functional anatomy of the genital tract with the creation of a neocervix. ${ }^{9}$

Many authors recommended hysterectomy for cervical ageniesis with a functioning uterine corpus to eliminate problem, such as sepsis, cryptomenorrhea, endometriosis and multiple surgeries. ${ }^{8}$

Whether, the patient desires definitive treatment with a hysterectomy or she wants to pursue a patent outflow tract and the possibility of future childbearing, evidencebased medicine must become the source for the surgical strategies. ${ }^{7}$

The development of extreme anxiety about the patient's femininity and a distortion of her physical image can affect her self-esteem, cause disharmony to her marital life. The psychological adjustment and general attitude are also very important in deciding as to what procedure should be used and when it should be done.

\section{REFERENCES}

1. Suganuma N, Furuhashi M, Moriwaki T, Tsukahara S, AndoT, Ishihara Y. Management of missed abortion in a patient with congenital cervical atresia. Fertil Steril 2002;77(5):1071-1073.

2. The American Fertility Society. The American Fertility Society classifications of adnexal adhesions, distal tubal occlusion, tubal occlusion secondary to tubal ligation, tubal pregnancies, Mullerian anomalies and intrauterine adhesions. Fertil Steril 1988;49(6):944-955.

3. Deffarges JV, Haddad B, Musset R, Paniel BJ. Uterovaginal anastomosis in women with uterine cervix atresia: long-term follow-up and reproductive performance. A study of 18 cases. Hum Reprod 2001;16(8):1722-1725.

4. Rock JA, Schlaff WD, Zacur HA, Jones HW Jr. The clinical management of congenital absence of the uterine cervix. Int J Gynaecol Obstet 1984;22(3):231-235.

5. Hampton HL. Role of the gynecologic surgeon in the management of urogenital anomalies in adolescents. Curr Opin Obstet Gynecol 1990;2(6):812-818.

6. Grimbizis GF, Tsalikis T, Mikos T, Papadopoulos N, Tarlatzis BC, Bontis JN. Successful end-to-end cervicocervical 
anastomosis in a patient with congenital cervical fragmentation: case report. Hum Reprod 2004;19(5):1204-1210.

7. Casey AC, Laufer MR. Cervical agenesis: septic death after surgery. Obstet Gynecol 1997;90(4 pt 2):706-707.

8. Lee CL, Wang CJ, Swei LD, Yen CF, Soong YK. Laparoscopic hemi-hysterectomy in treatment of a didelphic uterus with a hypoplastic cervix and obstructed hemivagina. Hum Reprod 1999;14(7):1741-1743.

9. Lee CL, Wang CJ, Liu YH, Yen CF, Lai YL, Soong YK. Laparoscopically assisted full thickness skin graft for reconstruction in congenital agenesis of vagina and uterine cervix. Hum Reprod 1999;14(4):928-930.
10. Buttram VC Jr, Gibbons WE. Mullerian anomalies: a proposed classification. (An analysis of 144 cases). Fertil Steril 1979; 32(1):40-46.

11. CreightonSM, Devis MC, Curter A. Laparoscopic management of cervical agenesis. Fertil Steril 2006;85(5):1510-1515.

12. Roberts CP, Rock JA. Surgical method in the treatment of congenital anomaly of uterine cervix: Current Opinion in Obstetrics and Gynecol 2011;23(4):251-257.

13. Khatoon H, Mahmud N, Chowdhury TA. Primary Amenorrhoea- analysis of 108 cases: South Asian Federation of Obstetrics and Gynecology 2010;2(2):105-108. 